\title{
Temporal immunological marker risk model for predicting severity of COVID-19 outcomes: early risers, late bloomers and general giants
}

\author{
Authors: Ramya Sriskandarajah, ${ }^{\mathrm{A}}$ Sruthi Ramaraju ${ }^{\mathrm{A}}$ and Ananya Diddapur ${ }^{\mathrm{A}}$
}

\section{Introduction}

Treatment strategies for severe COVID-19 presentations have improved. However, predicting severity early remains a challenge, as the onset of deterioration is highly variable and difficult to recognise. Patients showing late deterioration are particularly affected by limited treatment options.

\section{Objectives}

> To temporally compare immunological 'phenotypes' of severe and non-severe COVID-19 presentations.

> To create a clinical tool to help clinicians stratify high-risk subgroups.

\section{Methods}

A literature search for temporally-mapped immunological markers showing differences between mild and severe COVID-19 patients was conducted on the PubMed database and results were pooled. Post-analysis, the biomarkers were split into three categories, according to when significant differences between mild and severe cases arose post-disease onset: 'early risers' ( $<7$ days), 'late bloomers' ( $>7$ days) and 'general giants' (whole disease course).

\section{Results and discussion}

The early risers chosen were IL-6, aspartate aminotransferase (AST) and creatine kinase-MB (CK-MB). For late bloomers, serum D-dimer, cardiac troponin and serum creatinine were selected. For general giants, neutrophils, lymphocyte count and serum ferritin were predictive (Fig 1).

IL-6 was significantly raised in severe versus mild COVID-19 patients, likely due to its role in cytokine storms, a key contributor to multi-organ failure and acute respiratory distress. Further, IL-6 levels correlate to pre-existing risk factors of severe disease, eg hypertension. ${ }^{1-3}$

Author: ${ }^{\mathrm{A}}$ Imperial College London, London, UK
AST levels also demonstrated a high correlation to COVID-19 mortality risk and strongly predicted systemic inflammation, and liver injury via direct viral invasion of cholangiocytes. ${ }^{4,5}$ Interestingly, CK-MB rose early in the disease course before returning to normal range, though levels were still higher in severe patients than mild $(\mathrm{p}<0.05) .{ }^{4} \mathrm{CK}-\mathrm{MB}$ is a known predictor of cardiac damage through direct invasion of cardiomyocytes via ACE2 receptor. ${ }^{6}$

The late bloomers, D-dimer, troponin and creatinine were all markedly increased in severe patients from day 7 onwards and were associated with coagulopathy, cardiac disease and renal impairment respectively. Subsequently, these patients had poorer outcomes with higher mortality rates. ${ }^{1,4,7-9}$

For general giants, severe cases presented with higher neutrophil and lower lymphocyte counts compared to mild, confirming the potential of neutrophil-lymphocyte ratio as a predictor of disease severity (Fig 2). ${ }^{1,2,4,7,10}$

High serum ferritin levels were associated with development of ARDS and cytokine storm, suggesting this marker may indicate a transition towards hyper-inflammatory state in severe COVID-19 cases. ${ }^{1}$

\section{Conclusion}

Our study compiles key immunological markers and their temporal importance in COVID-19 disease progression. A comprehensive timescale of these markers can alert clinicians to potential deterioration in advance, and guide management and treatment options. Used in conjunction with patient characteristics and presentation, our study provides an efficient and simple method of streamlining risk stratification.

\section{Conflicts of interest}

None declared. 
Temporal immunological marker risk model for predicting severity of COVID-19 outcomes: early risers, late bloomers and general giants

IL6

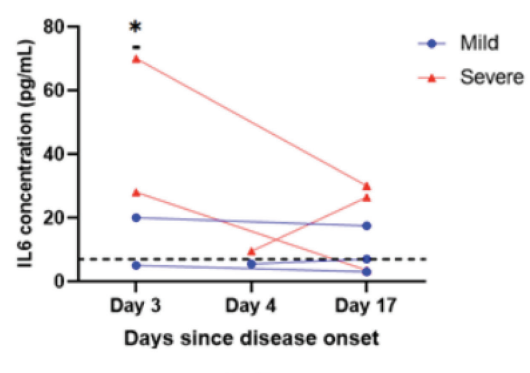

D-dimer

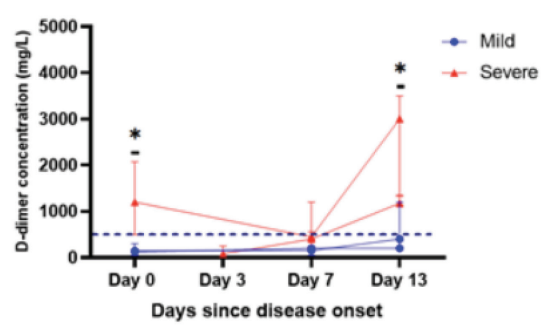

Neutrophils

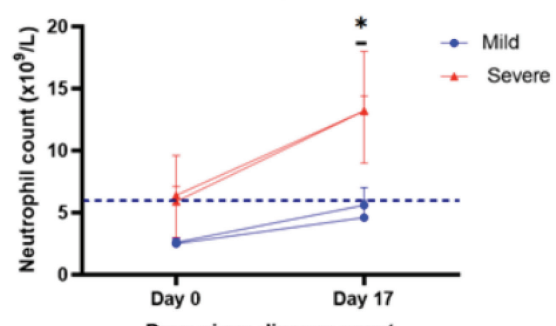

Days since disease onset
Aspartate aminotransferase

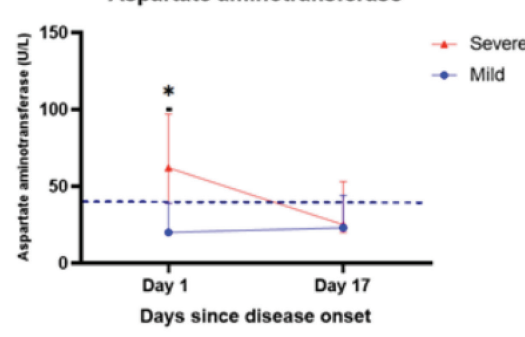

Serum creatinine

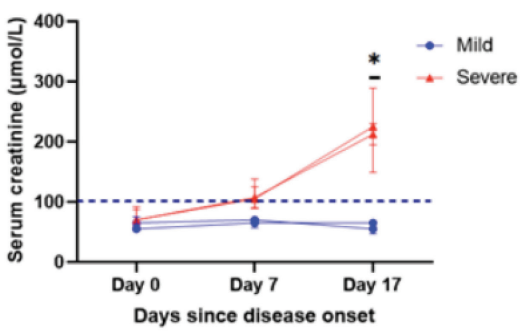

Lymphocyte count

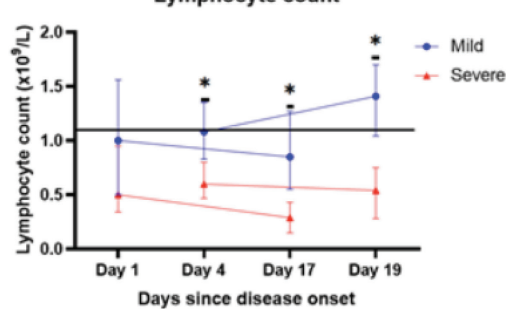

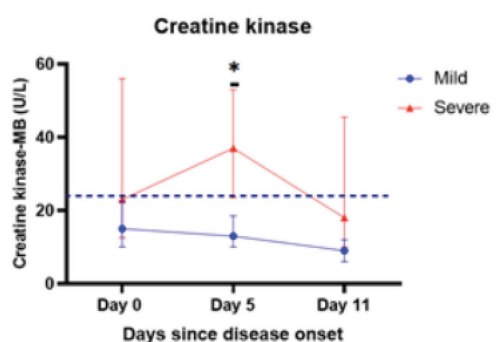

Cardiac troponin

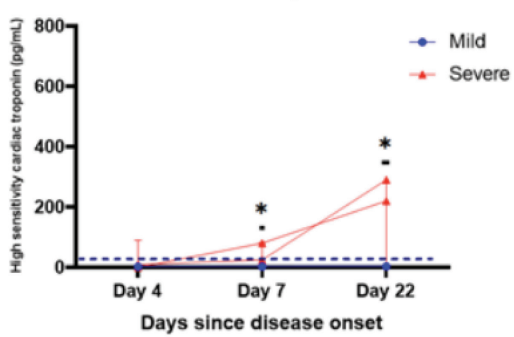

Serum ferritin

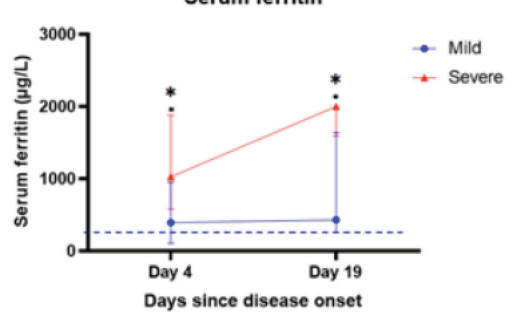

Fig 1. Line graph summarising predictors through disease course. ${ }^{*}$ Statistically significant $(p<0.05)$ points are shown by the asterisk. The normal range upper limit of each parameter is represented by the dashed blue line. The black solid line represents the lower limit of the lymphocyte count. ${ }^{1-5,7-10}$

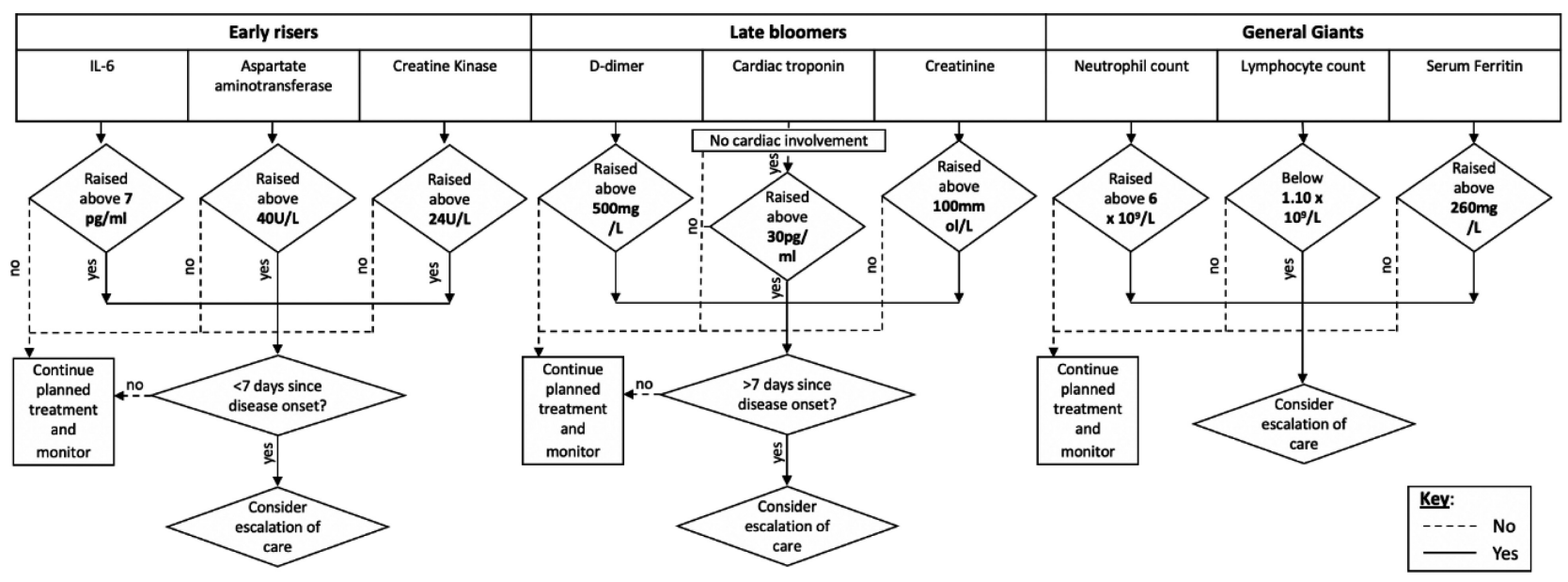

Fig 2. Example flowchart as a clinical tool to distinguish severe COVID-19 cases. 


\section{References}

1 Zhou F, Yu T, Du R et al. Clinical course and risk factors for mortality of adult inpatients with COVID-19 in Wuhan, China: a retrospective cohort study. Lancet 2020;395:1054-62.

2 Liu J, Li S, Liu J et al. Longitudinal characteristics of lymphocyte responses and cytokine profiles in the peripheral blood of SARS-CoV-2 infected patients. Lancet EBioMedicine 2020;55:23523964.

3 Zhu Z, Cai T, Fan L et al. Clinical value of immune-inflammatory parameters to assess the severity of coronavirus disease 2019. Int J Infect Dis 2020;95:332-9.

4 Wang D, Yin Y, Hu C et al. Clinical course and outcome of 107 patients infected with the novel coronavirus, SARS-CoV-2, discharged from two hospitals in Wuhan, China. Critical Care 2020;24:188.

5 Lei F, Liu YM, Zhou F et al. Longitudinal association between markers of liver injury and mortality in COVID-19 in China. Hepatology 2020;72:389-98.
6 Li L, Zhou Q, Xu J. Changes of laboratory cardiac markers and mechanisms of cardiac injury in coronavirus disease 2019. Biomed Res Int 2020;2020:7413673.

7 Wang D, Hu B, Hu C et al. Clinical characteristics of 138 hospitalised patients with 2019 novel coronavirus-infected pneumonia in Wuhan, China. JAMA 2020;323:1061-9.

8 Fu J, Kong J, Wang W et al. The clinical implication of dynamic neutrophil to lymphocyte ratio and D-dimer in COVID-19: A retrospective study in Suzhou China. Thrombosis Research 2020;192: 3-8.

9 Deng Q, Hu B, Zhang Y et al. Suspected myocardial injury in patients with COVID-19: Evidence from front-line clinical observation in Wuhan, China. Int J Cardiol 2020;311:116-21.

10 Zheng $\mathrm{Y}$ et al. The hemocyte counts as a potential biomarker for predicting disease progression in COVID-19: a retrospective study. Clin Chem Lab Med 2020;58:1106-15. 